\title{
Coupe préparatoire et croissance en surface terrière d'une sapiniére de seconde venue à la forêt modèle du Bas-Saint-Laurent, Québec
}

\author{
par Richard Zarnovican ${ }^{1}$, Jean-Martin Lussier ${ }^{1}$ et Claude Laberge ${ }^{1}$
}

\begin{abstract}
Dans le cadre de travaux sur la régénération naturelle par coupe progressive, une étude a été réalisée sur la croissance du sapin baumier (Abies balsamea [L.] Mill.) en surface terrière 5 ans après la coupe préparatoire. L'expérience a été menée dans une sapinière à bouleau jaune de seconde venue âgée de 60 ans. Le dispositif expérimental comprenait 16 placettes, dont un témoin et trois traitements d'éclaircie par le bas (15, 30 et $45 \%$ de la surface terrière enlevée). Dans les placettes, les arbres ont réagi à l'éclaircie par un taux d'accroissement en diamètre moyen de 6 à $12 \%$ selon l'intensité de l'intervention. La surface terrière des placettes présente un taux d'accroissement périodique moyen de 7,4\%, indépendamment de l'intensité du traitement. De fortes corrélations ont été établies entre certaines caractéristiques de la cime verte de l'arbre et son accroissement périodique en surface terrière. Cet accroissement dépend fortement du dhp au début de la période et de l'intensité du traitement. Les arbres de taille relative supérieure sont plus productifs que les autres et l'effet de la coupe sur l'accroissement pérodique en surface terrière par rapport aux témoins est significatif lorsque son intensité est supérieure à 30\%. Par contre, le ratio de l'accroissement périodique en surface terrière après la coupe sur l'accroissement périodique avant la coupe ne dépend que de l'intensité du traitement. Enfin, l'intensité de la coupe n'a pas influencé l'accroissement périodique en surface terrière par mètre carré de la cime verte.
\end{abstract}

Mots-clés : coupe préparatoire, croissance en surface terrière, sapin baumier

Balsam fir basal area growth was studied 5 years after the preparatory cut in the context of natural regeneration by the shelterwood system. The study was carried out in a 60-year-old second-growth balsam fir-yellow birch stand. The felling trials were realized in 16 plots and consisted of control and three felling regimes $(15,30$ and $45 \%$ of removed basal area). The periodic (5 years) mean growth rate on dbh of trees in plots varies between 6 and $12 \%$ in response to felling intensity. The basal area of plots presents a mean periodic growth rate of $7.4 \%$ independently of felling intensity. There are highly significant correlations between the live crown characteristics and periodic basal area growth. This growth is highly correlated with inital diameter and intensity of felling. The trees of higher relative size are more productive than the others and the impact of felling on periodic basal area growth is significant when the intensity of felling is greater than $30 \%$. Conversely, the ratio between periodic basal area growth after treatment and periodic basal area growth before treatment is correlated only with the intensity of felling. Finally, intensity of felling had no effect on periodic basal area growth per square meter of crown projected area.

Key words: preparatory felling, basal area growth, balsam fir

\section{Introduction}

Dans le cadre de l'aménagement équienne, la coupe progressive est considérée comme une méthode de choix pour la conduite de peuplements forestiers (Matthews 1994). En effet, grâce à sa flexibilité de façonner les peuplements selon les exigences de la plupart des essences, cette méthode permet d'assurer le renouvellement naturel de plusieurs types forestiers de l'est du Canada

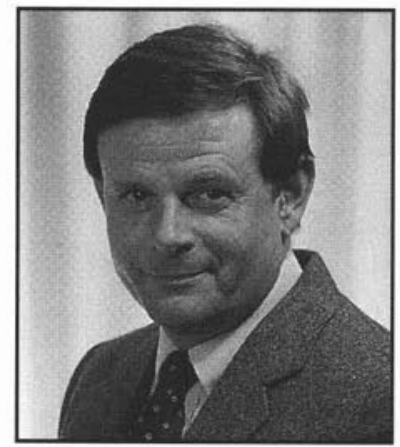

Richard Zarnovican (Hannah 1988). La coupe progressive vise à assurer l'éducation et la régénération des peuplements. L'éducation est réal-

${ }^{1}$ Ressources naturelles Canada, Service canadien des forêts, Centre de foresterie des Laurentides, 1055, rue du P.E.P.S., C.P. 3800, Sainte-Foy, Québec G1V 4C7.

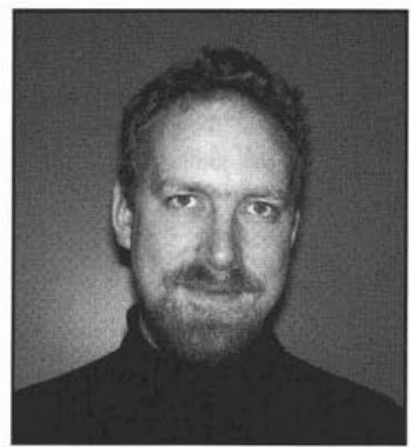

Jean-Martin Lussier

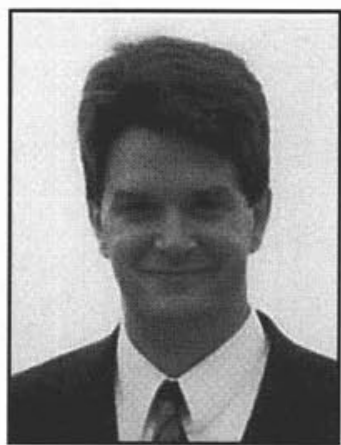

Claude Laberge isée dans les futaies mûres par les coupes préparatoires et son objectif est de constituer des semenciers bien formés, capables de produire des graines viables. La régénération du peuplement est réalisée par les coupes semencières pour constituer une réserve de semis et elle se termine par une coupe finale lorsque l'installation du jeune peuplement dans sa composition et sa densité est complètement terminée (Matthews 1994, Burschel et Huss 1997). 
Dans le cas des peuplements de seconde venue d'épinette blanche (Picea glauca [Moench] Voss) et de sapin baumier, plusieurs études ont expérimenté la coupe progressive pour assurer leur régénération naturelle (Baldwin 1977, Yuill 1980, Hannah 1988, Frank 1990, Youngblood 1990, Raymond 1998). La coupe progressive pratiquée lors de ces expériences se résume en deux, rarement en trois interventions. Généralement, la première intervention correspond à une coupe partielle, appelée aussi la coupe semencière, et la seconde à la coupe finale (Johnston 1986, Hannah 1988).

La coupe partielle est pratiquée pour stimuler la croissance des arbres résiduels et pour diminuer les coûts supplémentaires que la coupe progressive engendre par rapport à la coupe à blanc (Burschel et Huss 1997). Les coûts supplémentaires de la coupe progressive pourraient être partiellement compensés par l'augmentation de la taille et de la qualité moyenne des tiges résiduelles. Ainsi, au Nouveau-Brunswick, après une coupe partielle de $40 \%$ de la surface terrière dans une sapinière à épinette blanche de 50 ans, Baldwin (1977) a rapporté un accroissement en surface terrière de $2,3 \%$ après cinq ans et de $10,2 \%$ après 10 ans. Après une coupe partielle de plus de $50 \%$ de la surface terrière, Corriveau (1971b) a conclu que les interventions aussi intenses ne sont pas appropriées à la conduite de riches sapinières équiennes au centre du Québec. En NouvelleAngleterre, après une coupe partielle dans un peuplement d'épinette blanche et de sapin baumier de 75 ans, Frank (1973) a conclu que ce genre d'intervention doit libérer la cime sur trois et préférablement quatre côtés avant que l'on puisse espérer avoir des effets significatifs sur la croissance radiale. Youngblood (1990) a établi que la croissance en surface terrière des arbres individuels augmente de 26,8\% par rapport au $16,5 \%$ des arbres témoins 14 ans après une coupe d'ensemencement dans une pessière blanche de 174 ans en Alaska. Enfin, en Saskatchewan, Steneker (1974) n'a trouvé aucune réaction d'une pessière blanche de 80 ans à une coupe partielle.

Dans le domaine de la sapinière à bouleau jaune du Québec, il existe peu d'information concernant la coupe progressive et ses effets sur la croissance en surface terrière et sur le développement des arbres résiduels. Dans cette perspective, une expérience avec la coupe préparatoire a été réalisée dans une sapinière de seconde venue à la forêt modèle du Bas-Saint-Laurent. L'objectif de cette expérience était d'évaluer les effets de la coupe préparatoire sur la croissance en surface terrière des arbres résiduels cinq ans après le traitement et de les comparer aux données des placettes témoins.

Les objectifs spécifiques sont (1) d'évaluer l'impact de la coupe préparatoire sur le dhp moyen, la surface terrière, la mortalité des placettes et l'état de la cime verte des arbres, (2) de comparer l'état de la cime verte entre les placettes éclaircies et les placettes témoins, (3) d'établir le degré de dépendance entre les caractéristiques de la cime verte et l'accroissement quinquennal en surface terrière des arbres et (4) de quantifier les effets de l'intensité de la coupe sur le rendement des tiges en surface terrière cinq ans après le traitement.

\section{Matériel et méthode \\ Peuplement}

La présente étude a été effectuée dans une sapinière de seconde venue à la forêt modèle du Bas-Saint-Laurent (lat. $48^{\circ}$ $17^{\prime} \mathrm{N}$, long. $67^{\circ} 50^{\prime} \mathrm{O}$, alt. $310 \mathrm{~m}$ ). Le peuplement est une futaie régulière de 60 ans dominée par le sapin baumier et il est issu d'une coupe à blanc des années 40 (Hatcher 1963). Le peuplement est caractérisé par une densité de 1590 tiges à l'hectare, une surface terrière de $35,6 \mathrm{~m}^{2} \cdot \mathrm{ha}^{-1}$, un volume marchand résineux de $260 \mathrm{~m}^{3} \cdot \mathrm{ha}^{-1}$ et une hauteur moyenne des dominants et codominants de $16,5 \mathrm{~m}$. Selon l'indice de site et la production, le peuplement se compare à la $1^{\text {ère }}$ classe de tables de Vézina et Linteau (1968).

Selon la composition des strates inférieures, le massif fait partie de la portion méridionale du domaine de la bétulaie jaune à sapin (Grandtner 1966, Thibault 1986). Il est situé au milieu et en bas d'une pente moyenne (16\%) exposée au sud-ouest. Le dépôt de surface est un till modérément à bien drainé, à texture loameuse. Le climat est subpolaire et subhumide continental (Proulx et al. 1987). La température moyenne annuelle est de $2,3^{\circ} \mathrm{C}$ et les précipitations annuelles sont de $950 \mathrm{~mm}$.

Le massif a subi des épidémies de la tordeuse des bourgeons de l'épinette (Choristoneura fumiferana [Clem.]) (Hatcher 1963) et deux importantes réductions de croissance du sapin baumier ont eu lieu entre 1949 et 1964 et entre 1971 et 1985 (Zarnovican et Laberge 1998). Dans les gros diamètres, le sapin baumier présente un taux élevé de carie rouge (Zarnovican 1998). La présence de la carie suggère d'appliquer des rotations sanitaires raccourcies et aussi de favoriser d'autres essences moins vulnérables comme l'épinette blanche.

\section{Dispositif expérimental}

L'objectif de la coupe préparatoire est d'établir une réserve des semenciers produisant des graines viables sans créer d'ouvertures excessives dans le peuplement ou d'envahissement du parterre par la végétation concurrentielle. Considérant les risques de chablis dans la sapinière (Linteau 1957, Johnston 1986), quatre intensités de coupe ont été comparées, soit 0,15 , 30 et $45 \%$, évaluées par le rapport $g_{c} / g_{a}$ (la surface terrière enlevée sur la surface terrière avant le traitement). Le dispositif expérimental est un plan entièrement aléatoire, constitué de 16 unités expérimentales contigües de $900 \mathrm{~m}^{2}$, chacune séparée par un sentier de débardage large de $3 \mathrm{~m}$. À chaque unité expérimentale a été assigné aléatoirement l'une des quatre intensités de coupe. Les mesures ont été réalisées dans une placette de $400 \mathrm{~m}^{2}$ établie au centre de chaque unité expérimentale.

Compte tenu du fait qu'il s'agit d'un peuplement non éduqué, les intensités d'interventions obtenues ne sont pas égales aux rapports visés et elles varient entre 12,3 et $46,9 \%$ selon la placette (Tableau 1). Selon le rapport $\mathrm{dhp}_{\mathrm{c}} / \mathrm{dhp}_{\mathrm{t}}$ (le dhp moyen des tiges coupées sur le dhp moyens des tiges avant la coupe) qui se situe entre 0,68 et 0,88 (Tableau 1), l'intervention s'apparente à une éclaircie par le bas, la coupe étant concentrée sur les arbres des strates sociales inférieures. Cette nature de coupe a été choisie car les arbres des strates inférieures sont moins efficaces en tant que semenciers et sont également moins efficaces pour réagir à la coupe de mise en lumière en termes d'accroissement en volume (Assmann 1970, Johnston 1986, Gilmore et Seymour 1996). La coupe a été réalisée à l'aide de scies mécaniques en août et septembre 1994 et le bois a été vidangé sur les sentiers de débusquage à la main.

\section{Données récoltées}

Dans les 12 placettes traitées, un inventaire des tiges a été effectué en septembre 1994 avant et après le traitement sylvicole et en septembre 1999. Les tiges vivantes ont été numérotées et marquées à la hauteur de $1,3 \mathrm{~m}(\mathrm{dhp})$ pour la prise de 


\begin{tabular}{|c|c|c|c|c|c|c|c|c|c|c|c|c|c|}
\hline Placette & $\begin{array}{c}\text { Coupe } \\
(\%)\end{array}$ & $\begin{array}{c}\mathrm{DHP}_{\mathrm{a}} \\
(\mathrm{cm})\end{array}$ & $\begin{array}{l}\mathrm{DHP}_{\mathrm{c}} \\
(\mathrm{cm})\end{array}$ & $\begin{array}{l}\mathrm{DHP}_{\mathrm{r}} \\
(\mathrm{cm})\end{array}$ & $\begin{array}{l}\operatorname{DHP}_{\mathrm{f}} \\
(\mathrm{cm})\end{array}$ & $\underset{\left(\mathbf{d m}^{2}\right)}{\mathbf{G}_{\mathrm{a}}}$ & $\begin{array}{c}\mathbf{G}_{\mathrm{r}_{2}} \\
\left(\mathbf{d m}^{2}\right)\end{array}$ & $\begin{array}{c}\mathbf{G}_{\mathbf{m}_{9}} \\
\left(\mathbf{d m}^{2}\right)\end{array}$ & $\begin{array}{c}\mathrm{G}_{\mathrm{f}} \\
\left(\mathbf{d m}^{2}\right)\end{array}$ & $\begin{array}{l}P_{G} \\
(\%)\end{array}$ & $\begin{array}{c}\mathbf{G}_{\mathrm{c}} / \mathbf{G}_{\mathrm{a}} \\
(\%)\end{array}$ & $\begin{array}{l}\text { DHP }{ }^{\prime} \\
\text { DHPa }\end{array}$ & $\begin{array}{c}\text { Mortalité } \\
(\%)\end{array}$ \\
\hline 11 & 15 & 14,4 & 9,8 & 15,8 & 17,0 & 154 & 135 & 4 & 147 & 8,9 & 12,3 & 0,68 & 3,0 \\
\hline 9 & 15 & 14,7 & 10,1 & 16,0 & 16,8 & 167 & 146 & 11 & 155 & 6,2 & 12,3 & 0,69 & 7,5 \\
\hline 3 & 15 & 15,4 & 11,0 & 16,5 & 17,5 & 156 & 135 & 12 & 142 & 5,2 & 13,1 & 0,72 & 8,9 \\
\hline 12 & 15 & 14,1 & 9,7 & 15,8 & 16,7 & 168 & 143 & 13 & 148 & 3,5 & 14,9 & 0,68 & 9,1 \\
\hline 10 & 30 & 14,0 & 10,7 & 16,0 & 17,4 & 135 & 101 & 0 & 119 & 17,8 & 24,9 & 0,76 & 0,0 \\
\hline 2 & 30 & 14,9 & 11,4 & 17,2 & 19,0 & 137 & 102 & 6 & 113 & 10,8 & 25,4 & 0,77 & 5,9 \\
\hline 1 & 30 & 16,4 & 13,5 & 18,4 & 20,2 & 144 & 101 & 12 & 106 & 5,0 & 29,9 & 0,82 & 11,9 \\
\hline 7 & 30 & 15,5 & 12,6 & 18,5 & 20,0 & 156 & 97 & 4 & 107 & 10,3 & 37,5 & 0,82 & 4,1 \\
\hline 6 & 45 & 15,4 & 13,5 & 17,6 & 19,4 & 151 & 88 & 21 & 80 & $-9,1$ & 41,9 & 0,87 & 23,9 \\
\hline 4 & 45 & 14,6 & 12,3 & 17,1 & 19,1 & 160 & 92 & 12 & 97 & 5,4 & 42,1 & 0,85 & 13,0 \\
\hline 5 & 45 & 14,5 & 12,8 & 16,6 & 18,7 & 137 & 74 & 4 & 85 & 14,9 & 46,0 & 0,88 & 5,4 \\
\hline 8 & 45 & 14,9 & 13,0 & 17,4 & 19,4 & 162 & 86 & 10 & 95 & 10,5 & 47,1 & 0,87 & 11,6 \\
\hline
\end{tabular}

Où : DNP - diamètre moyen à 1,3 m; G - surface terrière; $P_{G}$ - taux d'accroissement en surface terrière entre 1994 et 1999 ; $a$ - avant traitement; $r$ - immédiatement après coupe; $\mathrm{c}$-coupe; mo-mortalité entre 1994 et 1999; f-en 1999.

mesures. Les mesures sur le diamètre à $1,3 \mathrm{~m}$ avec écorce $\left(\mathrm{dhp}_{\mathrm{ae}}\right)$ selon l'essence ont été recueillies au millimètre près à l'aide d'un compas forestier. En septembre 1999, en plus des 12 placettes traitées, nous avons également inventorié les quatre placettes témoins. Au cours de l'inventaire de 1999, la compression latérale des cimes de tous les sapins a été évaluée visuellement par les cotes $0,25,50,75$ et $100 \%$ et ce aussi bien dans les placettes témoins que dans les placettes traitées. La compression latérale, déterminée par le nombre de côtés comprimés de cime verte, correspond à la réduction de la taille de cime par rapport à une tige d'une grosseur comparable croissant à découvert.

Considérant l'importance du sapin baumier sur la surface terrière du massif, la présente étude porte sur le sapin baumier seulement. Parmi les arbres des placettes de 1999, nous avons choisi au hasard 10 arbres échantillons par placette traitée et 20 arbres échantillons par placette témoin pour construire des mesures composites pour chaque unité expérimentale (placette). Sur l'arbre, la croissance radiale a été établie sur la carotte prélevée au dhp selon la procédure décrite par Noël et Rouleau (1979), en mesurant l'accroissement radial des 10 et 5 dernières années, ainsi que l'épaisseur de l'écorce. Ces mesures ont été réalisées à l'aide d'un compas électronique au $0,01 \mathrm{~mm}$ près. L'accroissement en surface terrière $\left(\mathrm{i}_{\mathrm{g}}\right)$ a été calculé à l'aide de la formule [1] :

$$
\mathrm{i}_{\mathrm{g}}=\pi \cdot \mathrm{i}_{\mathrm{r}}\left(\mathrm{dhp}_{\mathrm{f}}-\mathrm{i}_{\mathrm{r}}\right)
$$

où $\mathrm{i}_{\mathrm{r}}$ est l'accroissement radial et $\mathrm{dhp}_{\mathrm{f}}$ est le dhp de l'arbre à la fin de la période.

La hauteur totale de l'arbre ainsi que la hauteur de la cime verte (hc) des tiges ont été mesurées à l'aide du dendromètre Haglof Vertex au décimètre près. Pour établir les caractéristiques des cimes, nous nous sommes inspirés de la procédure proposée par Dong et Kramer (1986). Le rayon de la cime verte a été mesuré aux quatre points cardinaux, à l'aide d'un plombeur optique (Dong et Kramer 1986) au décimètre près. La distance horizontale entre l'arbre échantillon et son voisin le plus proche a été mesurée à l'aide d'un galon au centimètre près. En plus de la distance, l'azimut du voisin et son dhp ont été notés.

La projection des cimes (PCV) a été établie en utilisant la moyenne de quatre rayons, alors que ce rayon moyen (rc) et la hauteur de la cime verte ont été utilisés pour calculer la sur- face latérale (SCV) et le volume de la cime verte (VCV). Considérant que la cime du sapin a une forme conique (Zarnovican 1982), la surface latérale et le volume de la cime verte ont été calculés à l'aide des formules pour le cône de révolution $[2,3]$.

$$
\begin{aligned}
& \mathrm{SCV}=\pi \cdot \mathrm{rc}\left(\mathrm{rc}^{2}+\mathrm{hc}^{2}\right)^{0,5} \\
& \mathrm{VCV}=\left(\pi \cdot \mathrm{rc}^{2} \cdot \mathrm{hc}\right) / 3
\end{aligned}
$$

Enfin, les dhp relatifs des arbres échantillons ont été calculés par rapport au dhp moyen de la placette de 1999 ( $\mathrm{dhp}_{\mathrm{i}} / \mathrm{dhp}_{\text {moy }}$ ) dans le but d'établir les classes de taille relative qu'on peut assumer aux classes sociales (Horne et al. 1986). Les principales statistiques de ces variables mesurées sur les arbres sont aux tableaux 2 et 3 .

\section{Analyse des données État de la cime verte}

On reconnaît que la vitesse de croissance n'est pas seulement influencée par la lumière, mais également par la compression latérale des cimes vertes (Assmann 1970, Frank 1973). Pour évaluer l'état de la cime verte, nous avons analysé l'ampleur de la compression latérale chez le sapin baumier à partir des 650 observations des 16 placettes d'étude.

À partir de ces mesures, un tableau croisé a été construit pour la compression de la cime avec trois modalités (faible $\leq 25 \%$, moyenne $>25 \leq 75 \%$, forte $>75 \%$ ), la taille relative de l'arbre dans le peuplement avec trois modalités (inférieure avec le dhp relatif $<0,90$, moyenne avec le dhp relatif $\geq 0,90$ et $<1,10$ et supérieure avec le dhp relatif $\geq 1,10$ ) et l'intensité de la coupe préparatoire avec quatre modalités (nulle pour le témoin, faible pour l'intensité entre 12,3 et $14,9 \%$, moyenne pour l'inensité de coupe entre 25,2 et $37,8 \%$ et la classe forte pour l'intensité entre 41,9 et $46,9 \%$ ).

Pour évaluer les relations entre ces variables, nous avons utilisé des modèles log - linéaires (Fienberg 1980). Ces modèles ont été ajustés par la procédure LOGLIN (SPSS Inc. 1998). La modélisation a été faite en partant du modèle complet pour enlever successivement les interactions d'ordre supérieur. La qualité de l'ajustement est déterminée à l'aide du test de $\chi^{2}$. Une petite valeur du $\chi^{2}$ est associée à un bon ajustement $(p>0,05)$. Le rejet d'un modèle $(p<0,05)$ implique qu'au moins 


\begin{tabular}{|c|c|c|c|c|c|c|c|c|c|c|c|c|c|c|c|}
\hline \multirow{2}{*}{ Placette } & \multirow{2}{*}{$\begin{array}{c}\text { Coupe } \\
(\%)\end{array}$} & \multicolumn{2}{|c|}{$\begin{array}{c}\mathrm{DHP}_{\text {ae }} \\
(\mathrm{cm})\end{array}$} & \multicolumn{2}{|c|}{$\begin{array}{l}\text { Hauteur } \\
\text { (m) }\end{array}$} & \multicolumn{2}{|c|}{$\begin{array}{l}\text { Distance-v } \\
\quad(\mathbf{m})\end{array}$} & \multicolumn{2}{|c|}{$\begin{array}{c}\mathrm{DHP}_{\mathrm{ae}}-\mathrm{v} \\
(\mathrm{cm})\end{array}$} & \multicolumn{2}{|c|}{$\begin{array}{c}\mathrm{ir}_{1} \\
(\mathrm{~mm})\end{array}$} & \multicolumn{2}{|c|}{$\begin{array}{c}\mathrm{ir}_{2} \\
(\mathrm{~mm})\end{array}$} & \multicolumn{2}{|c|}{$\underset{\left(\mathbf{d m}^{3}\right)}{\text { Volume }_{\text {ae }}}$} \\
\hline & & $\mathbf{x}$ & $\mathbf{s x}$ & $\mathbf{x}$ & $\mathbf{S X}$ & $\mathbf{x}$ & $\mathbf{S X}$ & $\mathbf{x}$ & $\mathbf{s x}$ & $\mathbf{x}$ & sx & $\mathbf{x}$ & $\mathbf{S X}$ & $\mathbf{x}$ & $\mathbf{s x}$ \\
\hline Tr-1 & 30 & 20,0 & 3,5 & 16,8 & 1,2 & 200 & 58 & 19,2 & 5,9 & 8,71 & 3,57 & 5,23 & 1,81 & 264 & 109 \\
\hline $\operatorname{Tr}-2$ & 30 & 16,7 & 2,8 & 15,3 & 1,5 & 160 & 27 & 21,9 & 4,2 & 4,73 & 2,87 & 3,56 & 1,88 & 173 & 66 \\
\hline $\operatorname{Tr}-3$ & 15 & 15,7 & 2,3 & 15,4 & 1,3 & 116 & 52 & 19,4 & 3,6 & 3,31 & 1,94 & 2,98 & 1,23 & 149 & 56 \\
\hline $\operatorname{Tr}-4$ & 45 & 17,8 & 2,8 & 15,8 & 1,4 & 220 & 38 & 19,4 & 2,9 & 6,96 & 3,03 & 3,94 & 2,13 & 200 & 74 \\
\hline $\operatorname{Tr}-5$ & 45 & 17,9 & 4,4 & 15,6 & 1,3 & 252 & 45 & 17,5 & 4,0 & 7,50 & 3,42 & 4,23 & 2,08 & 210 & 117 \\
\hline $\operatorname{Tr}-6$ & 45 & 19,3 & 4,3 & 15,3 & 1,2 & 244 & 37 & 19,5 & 4,3 & 7,24 & 1,56 & 3,07 & 1,39 & 249 & 133 \\
\hline $\operatorname{Tr}-7$ & 30 & 23,9 & 6,8 & 17,4 & 2,2 & 214 & 95 & 19,5 & 5,2 & 9,20 & 3,18 & 6,05 & 2,49 & 414 & 282 \\
\hline $\operatorname{Tr}-8$ & 45 & 17,7 & 4,4 & 15,9 & 2,2 & 197 & 66 & 18,3 & 6,3 & 8,43 & 3,24 & 4,24 & 1,42 & 206 & 113 \\
\hline Tr-9 & 15 & 16,1 & 2,8 & 15,8 & 0,9 & 103 & 41 & 16,1 & 3,3 & 4,06 & 1,91 & 3,13 & 1,68 & 158 & 59 \\
\hline $\operatorname{Tr}-10$ & 30 & 17,0 & 4,6 & 15,4 & 1,3 & 125 & 80 & 17,2 & 4,0 & 6,18 & 2,85 & 4,30 & 2,33 & 190 & 124 \\
\hline Tr-11 & 15 & 17,7 & 5,6 & 16,2 & 2,0 & 158 & 80 & 16,6 & 4,6 & 4,56 & 2,47 & 3,77 & 2,74 & 215 & 165 \\
\hline Tr-12 & 15 & 16,4 & 5,1 & 15,0 & 1,5 & 117 & 24 & 16,6 & 5,0 & 4,06 & 2,34 & 3,35 & 2,96 & 181 & 132 \\
\hline Té-1 & 0 & 18,7 & 2,9 & 16,6 & 1,6 & 135 & 67 & 18,7 & 5,9 & 4,73 & 1,75 & 3,95 & 1,54 & 226 & 79 \\
\hline Té-2 & 0 & 18,0 & 4,1 & 15,8 & 1,6 & 136 & 87 & 18,6 & 5,6 & 4,36 & 2,30 & 3,47 & 2,02 & 212 & 118 \\
\hline Té-3 & 0 & 15,8 & 4,2 & 15,0 & 1,7 & 130 & 57 & 15,1 & 3,5 & 3,86 & 2,61 & 3,63 & 2,87 & 161 & 109 \\
\hline Té-4 & 0 & 15,6 & 4,1 & 15,0 & 1,3 & 129 & 62 & 13,9 & 2,4 & 4,79 & 3,18 & 3,86 & 2,80 & 156 & 105 \\
\hline
\end{tabular}

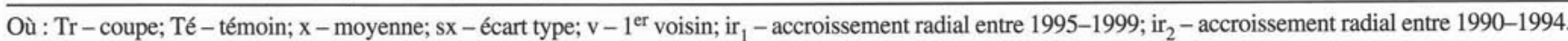

\begin{tabular}{|c|c|c|c|c|c|c|c|c|c|c|c|c|}
\hline \multirow[b]{2}{*}{ Placette } & \multicolumn{2}{|c|}{ Hauteur (m) } & \multicolumn{2}{|c|}{ Rayon (m) } & \multicolumn{2}{|c|}{ Compression (\%) } & \multicolumn{2}{|c|}{ Projection* $\left(\mathbf{m}^{2}\right)$} & \multicolumn{2}{|c|}{ Surface**(m²) } & \multicolumn{2}{|c|}{ Volume $\left(\mathbf{m}^{3}\right)$} \\
\hline & $\mathbf{x}$ & sx & $\mathbf{x}$ & $\mathbf{s x}$ & $\mathbf{x}$ & sx & $\mathbf{x}$ & $\mathbf{s x}$ & $\mathbf{x}$ & $\mathbf{s x}$ & $\mathbf{x}$ & $\mathbf{s x}$ \\
\hline $\operatorname{Tr}-1$ & 8,1 & 1,9 & 127,0 & 18,5 & 30 & 26 & 5,2 & 1,6 & 31,9 & 12,4 & 14,3 & 8,0 \\
\hline Tr-2 & 7,2 & 1,8 & 98,5 & 16,6 & 42 & 17 & 3,1 & 1,0 & 21,9 & 8,0 & 7,8 & 4,2 \\
\hline Tr-3 & 5,2 & 0,9 & 66,5 & 20,1 & 72 & 14 & 1,5 & 0,9 & 10,4 & 5,0 & 2,8 & 2,0 \\
\hline $\operatorname{Tr}-4$ & 6,4 & 1,7 & 106,7 & 21,0 & 35 & 21 & 3,7 & 1,6 & 21,8 & 11,6 & 8,5 & 7,0 \\
\hline $\operatorname{Tr}-5$ & 6,6 & 2,6 & 109,2 & 30,9 & 20 & 26 & 4,0 & 2,2 & 24,3 & 15,9 & 10,3 & 10,1 \\
\hline $\operatorname{Tr}-6$ & 7,4 & 1,4 & 121,7 & 18,1 & 15 & 21 & 4,8 & 1,4 & 28,6 & 7,0 & 11,8 & 4,2 \\
\hline $\operatorname{Tr}-7$ & 8,8 & 2,1 & 145,5 & 37,3 & 22 & 25 & 7,0 & 3,5 & 41,2 & 19,5 & 22,4 & 16,0 \\
\hline $\operatorname{Tr}-8$ & 7,6 & 2,1 & 116,3 & 25,0 & 37 & 29 & 4,4 & 1,7 & 28,6 & 12,2 & 12,1 & 6,7 \\
\hline Tr-9 & 6,7 & 2,1 & 86,5 & 37,9 & 57 & 33 & 2,8 & 1,9 & 18,8 & 10,8 & 7,0 & 5,4 \\
\hline Tr-10 & 6,8 & 2,4 & 112,1 & 35,9 & 42 & 31 & 4,3 & 2,8 & 24,9 & 19,1 & 11,4 & 12,9 \\
\hline Tr-11 & 6,4 & 2,3 & 106,1 & 43,8 & 50 & 35 & 4,1 & 3,5 & 23,9 & 20,4 & 11,1 & 14,8 \\
\hline Tr-12 & 5,6 & 2,1 & 92,1 & 29,7 & 60 & 24 & 2,9 & 1,9 & 17,2 & 11,5 & 6,4 & 6,2 \\
\hline Té-1 & 6,5 & 1,2 & 114,0 & 25,5 & 45 & 28 & 4,3 & 1,9 & 23,0 & 8,1 & 9,6 & 5,4 \\
\hline Té-2 & 6,6 & 2,3 & 107,5 & 36,1 & 51 & 26 & 4,0 & 2,4 & 22,8 & 13,5 & 10,0 & 8,2 \\
\hline Té-3 & 5,5 & 1,9 & 83,0 & 36,6 & 56 & 33 & 2,6 & 2,4 & 15,7 & 13,0 & 6,0 & 7,8 \\
\hline Té-4 & 5,4 & 1,5 & 90,1 & 36,5 & 46 & 42 & 3,0 & 2,2 & 16,3 & 9,4 & 5,9 & 4,9 \\
\hline
\end{tabular}

Où : $\mathrm{Tr}$ - coupe; Té - témoin; $\mathrm{x}$ - moyenne; sx - écart type; *projection verticale; ** surface latérale.

une interaction ou association significative est manquante pour ajuster adéquatement toutes les cellules du tableau.

L'étude des estimateurs de paramètres standardisés (le rapport entre l'estimateur du paramètre et l'écart type de cet estimateur) pour chaque cellule de tableau croisé permet alors d'approfondir l'étude des interactions et des associations. En effet, les cellules associées à un estimateur standardisé plus grand que 2 (ou plus petit que -2) sont associées à des interactions ou à des associations qui ne sont pas prises en compte par le modèle. Par exemple, l'étude avec et sans un terme d'interaction, en relation avec l'apparition de grands résidus standardisés, permet d'associer ce terme d'interaction avec les cellules mal ajustées, expliquant ainsi la signification du terme d'interaction.

\section{Accroissement quinquennal en surface terrière des arbres échantillions}

Dans le cas des arbres échantillons (10 arbres par placette traitée et 20 arbres par placette témoin), les relations entre l'accroissement quinquennal en surface terrière (ig - variable dépendante) et les variables explicatives qui caractérisent la dimension de l'arbre échantillon (dhp, hauteur), sa position relative dans la structure diamétrale de la placette $\left(\mathrm{dhp}_{\mathrm{i}} / \mathrm{dhp}_{\mathrm{moy}}\right)$, sa cime verte (hauteur, rayon, projection, surface, compression, volume) et l'espace disponible (distance au premier voisin et son dhp), ont été étudiées à l'aide des régressions linéaires simples. Ces relations ont été établies à l'aide du modèle linéaire de la forme générale ig $=\beta_{0}+\beta_{1} X$ afin de mieux comprendre les liens entre l'accroissement en surface terrière et les différentes composantes de l'arbre et de son espace de croissance.

\section{Intensité de coupe et croissance en surface terrière des placettes}

La relation entre l'intensité de la coupe préparatoire et l'accroissement quinquennal moyen des placettes en surface terrière a été évaluée par la régression multiple. Les variables indépendantes sont l'intensité de l'intervention dans la placette et le dhp moyen de la placette en 1994. Les variables dépendantes sont l'accroissement moyen en surface terrière des tiges résiduelles au cours des 5 années qui ont suivi le traite- 


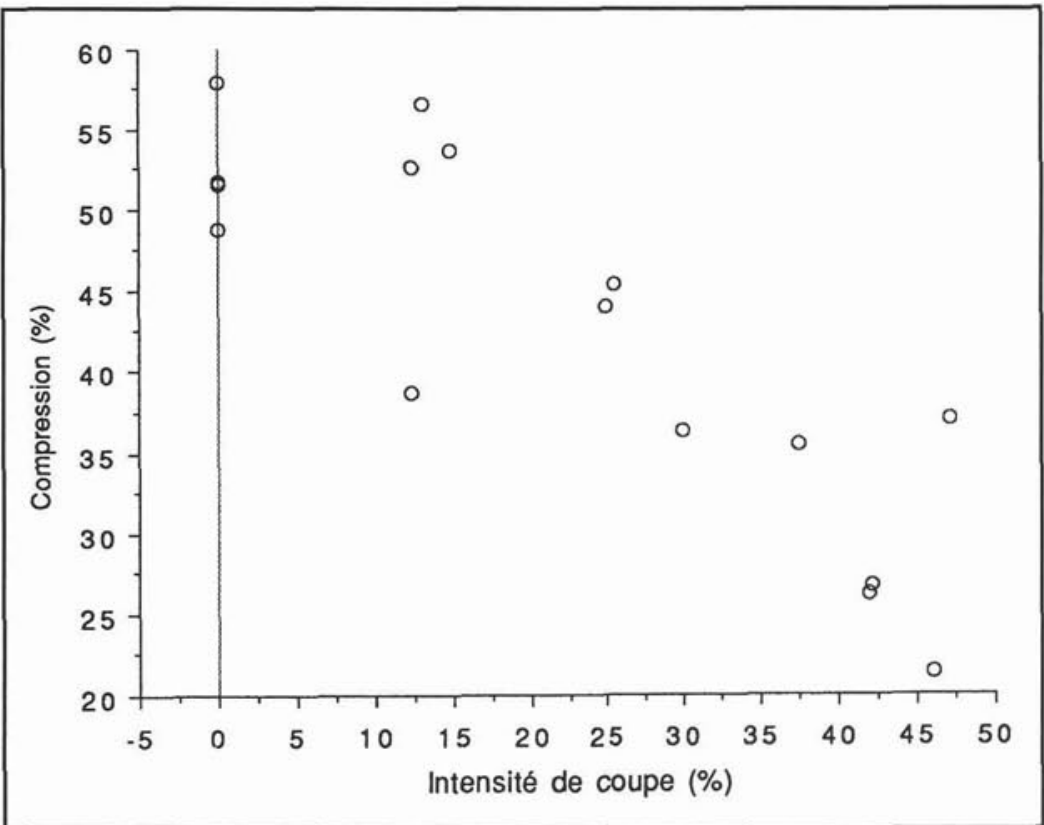

Fig. 1. Compression de la cime verte (moyenne par placette) selon l'intensité de coupe (témoin $=0 \%$ ).

ment (ig ${ }_{\mathrm{AP}}$ en $\mathrm{dm}^{2}$ ), le ratio moyen de l'accroissement en surface terrière des 5 années qui ont suivi le traitement par rapport aux 5 années qui l'ont précédé (ratio ig ${ }_{a p} / i_{\mathrm{AV}}$ ) et enfin, le rapport moyen entre ig ${ }_{\mathrm{AP}}$ et la surface de la projection de cime verte (ig/PCV).

\section{Résultats et discussion \\ Diamètre moyen des placettes}

La mise à distance des arbres lors d'une éclaircie stimule la croissance radiale du sapin baumier autant dans les jeunes peuplements (Lavigne et Donnelly 1989, Zarnovican et Laberge 1996) que dans les peuplements âgés (Hatcher 1961, Corriveau 1971a). En plus de l'effet positif sur la croissance radiale, on reconnaît également les effets de l'éclaircie sur la structure et sur la composition des peuplements (Marquis et Ernst 1991).

Les diamètres moyens des placettes (Tableau 1) ne sont pas statistiquement différents $(\mathrm{p}=0,07)$ avant la coupe préparatoire, mais ils le deviennent immédiatement après la coupe. En effet, l'intervention par le bas a produit une augmentation mathématique du diamètre moyen des placettes entre 8 et $18 \%$ par rapport à celui avant le traitement pour l'intensité du traitement qui varie de 12 à $47 \%$. Cinq ans après la coupe préparatoire, les arbres ont réagi à l'augmentation de l'espace disponible par un accroissement du diamètre moyen des placettes. Moindre que l'effet mathématique de 1994, le diamètre moyen des placettes, 5 ans après la coupe, s'est accru entre 6 et $12 \%$ selon l'intensité de l'intervention (Tableau 1). Ces résultats sont en accord avec les données de Corriveau (1971a) qui a obtenu, 5 ans aprés une éclaircie de 20 et de $30 \%$ dans une sapinière à Dryopteris de 40 ans, des taux un peu plus élevés de 8,5 et de $12,0 \%$.

\section{Surface terrière des placettes}

En 1999, 5 ans après la coupe préparatoire, le taux d'accroissement en surface terrière des placettes a atteint en moyenne $7,4 \%$ $\left(s_{x}=6,7 \%\right)$ et seulement une placette a subi une perte de l'ordre de $9 \%$ (Tableau 1). Cette perte provient de la mortalité accrue dans la placette 6. L'accroissement quinquennal en surface terrière des placettes traitées est indépendant de l'intensité du traitement ( $\mathrm{p}=0,99)$, ce qui indique que l'intensité du traitement n'a pas eu d'effet sur la croissance en surface terrière des placettes ni, par conséquent, sur la croissance en volume total.

Entre 1994 et 1999, les placettes ont subi des pertes en surface terrière par mortalité naturelle et par chablis. Pour cette période, le taux moyen de mortalité par placette est de $8,7 \%$ $\left(s_{x}=6,2 \%\right)$, avec une perte très importante de $23,9 \%$ dans la placette 6 . Le taux de mortalité par placette est statistiquement indépendant de l'intensité de la coupe $(\mathrm{p}=0,21)$. À titre de comparaison, 5 ans après une coupe partielle de $40 \%$, Corriveau (1971b) rapporte un taux de mortalité de $15 \%$ pour une sapinière à Drypteris-Oxalis de 50 ans et un taux de $26 \%$ pour une sapinière de 70 ans.

\section{État de la cime verte des sapins des placettes}

La compression des cimes vertes du sapin baumier est évaluée à partir des 650 observations prises dans 16 placettes d'étude. La compression moyenne de la cime verte est semblable entre les placettes-témoins (l'intensité du traitement est nulle) et les placettes dont l'intensité du traitement n'a pas dépassé $20 \%$ (Fig. 1). En revanche, pour les coupes plus intenses, la compression moyenne du sapin diminue avec l'intensité du traitement et avec la taille croissante de l'arbre (Fig. 2). Ces observations sont confirmées par l'analyse des tableaux de fréquences. En effet, les modèles log-linéaires indiquent qu'il y a une très forte association entre la compression et le traitment sylvicole $(\mathrm{p}<0,0001)$ et entre la compression et la taille relative ( $\mathrm{p}<0,0001)$. Dans le cas de l'association entre la compression et le traitement sylvicole (Tableau 4), les résidus standardisés (RS) indiquent qu'elle provient d'une faible proportion des tiges comprimées après la coupe forte (taille relative inférieure $[\mathrm{RS}=3,32]$ et moyenne $[\mathrm{RS}=2,97]$ ), mais également d'une forte proportion des cimes comprimées dans le témoin (taille relative inférieure $[\mathrm{RS}=2,88]$ et moyenne $[\mathrm{RS}=-2,01])$. 


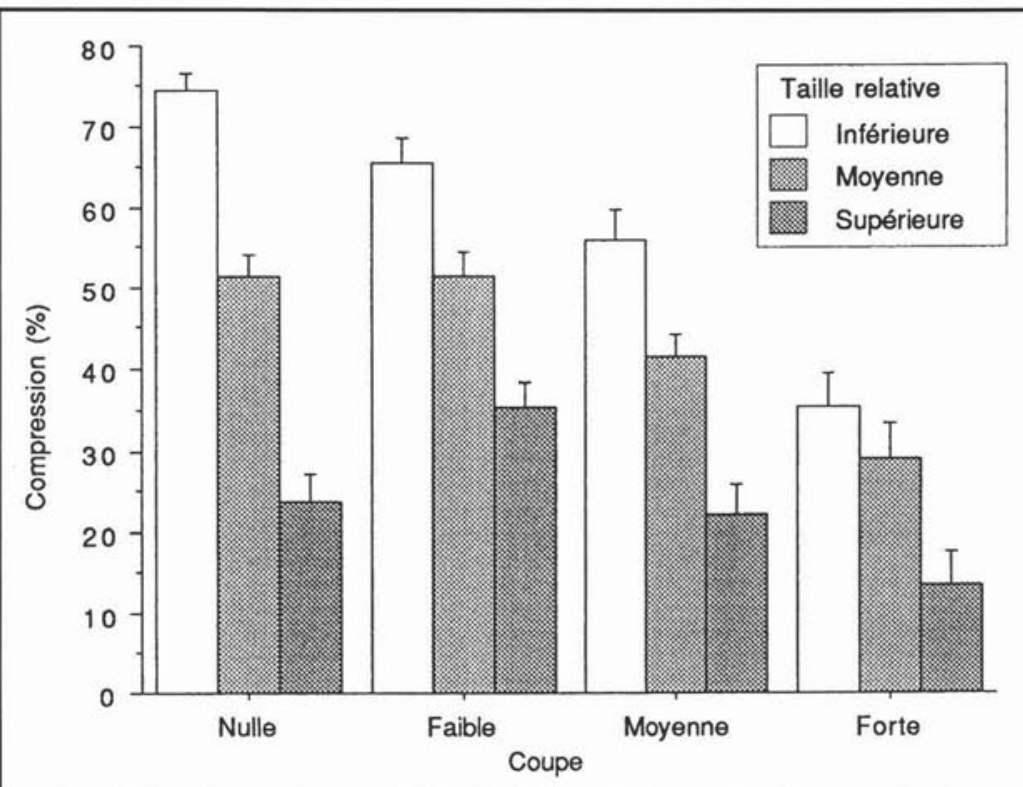

Fig. 2. Compression de la cime verte (moyenne \pm erreur type) selon l'intensité de coupe et la taille relative de l'arbre.

Tableau 4. Distribution des 650 sapins baumiers selon la compression de la cime verte, l'intensité de coupe et la taille relative de l'arbre

\begin{tabular}{|c|c|c|c|c|c|}
\hline \multirow[b]{2}{*}{ Compression } & \multicolumn{5}{|c|}{ Coupe } \\
\hline & Nulle & Faible & Moyenne & Forte & Total \\
\hline & & & Taille relative inférieure & & \\
\hline Faible & $6,6 \%$ & $8,8 \%$ & $21,4 \%$ & $35,0 \%$ & $14,5 \%$ \\
\hline Moyenne & $15,4 \%$ & $27,9 \%$ & $38,1 \%$ & $57,5 \%$ & $29,9 \%$ \\
\hline Forte & $78,0 \%$ & $63,2 \%$ & $40,5 \%$ & $7,5 \%$ & $55,6 \%$ \\
\hline \multirow[t]{2}{*}{ Total } & $100,0 \%(91 *)$ & $100,0 \%(68)$ & $100,0 \%(42)$ & $100,0 \%(40)$ & $100,0 \%(241)$ \\
\hline & & & Taille relative moyenne & & \\
\hline Faible & $18,6 \%$ & $30,5 \%$ & $33,3 \%$ & $61,1 \%$ & $32,4 \%$ \\
\hline Moyenne & $50,0 \%$ & $32,2 \%$ & $54,2 \%$ & $30,6 \%$ & $42,7 \%$ \\
\hline Forte & $31,4 \%$ & $37,3 \%$ & $12,5 \%$ & $8,3 \%$ & $24,9 \%$ \\
\hline \multirow[t]{2}{*}{ Total } & $100,0 \%(70)$ & $100,0 \%(59)$ & $100,0 \%(48)$ & $100,0 \%(36)$ & $100,0 \%(213)$ \\
\hline & & & Taille relative supérieure & & \\
\hline Faible & $62,3 \%$ & $45,5 \%$ & $75,0 \%$ & $76,9 \%$ & $59,7 \%$ \\
\hline Moyenne & $32,8 \%$ & $44,2 \%$ & $21,9 \%$ & $23,1 \%$ & $34,2 \%$ \\
\hline Forte & $4,9 \%$ & $10,4 \%$ & $3,1 \%$ & $0,0 \%$ & $6,1 \%$ \\
\hline Total & $100,0 \%(61)$ & $100,0 \%(77)$ & $100,0 \%(32)$ & $100,0 \%(26)$ & $100,0 \%$ (196) \\
\hline
\end{tabular}

*Effectif de la classe.

L'association significative entre la compression des cimes vertes et la taille relative provient d'un taux élevé des cimes vertes sans compression dans la classe supérieure (aucune coupe $[R S=5,56]$, coupe faible $[R S=2,67]$ et coupe moyenne $[\mathrm{RS}=3,04]$ ) et d'un taux également élevé des cimes vertes chétives et fortement comprimées dans la classe inférieure (témoin $[\mathrm{RS}=5,02]$, coupe faible $[\mathrm{RS}=3,75]$ et moyenne $[\mathrm{RS}=$ $2,95])$. Si le taux de compression des cimes vertes dans les tailles relatives moyennes et inférieures varie beaucoup d'une modalité de coupe à l'autre, il est en revanche beaucoup plus homogène pour la taille supérieure selon le test de $\chi^{2}$. Ainsi, les modifications dans la hiérarchie et la structure des placettes dues à la coupe se reflètent également dans l'état de la cime verte au niveau des classes sociales moyennes et inférieures, ce qui corrobore la nature de la coupe par le bas.

\section{Accroissement quinquennal en surface terrière des arbres échantillonnés}

Les résultats obtenus montrent que la coupe préparatoire stimule la croissance des arbres résiduels et augmente leur diamètre moyen. Cette réaction positive des arbres au traitement indique que le peuplement de 60 ans possède encore la capacité de réagir à l'augmentation de l'espace disponible et à l'amélioration des conditions de croissance. Pour mieux comprendre les liens entre l'accroissement quinquennal en surface terrière et certaines variables explicatives (dimensions, cime verte, espace disponible), les coefficients de détermination ont été calculés pour le modèle linéaire (Tableau 5). Les résultats exprimés pour les placettes traitées par la coupe préparatoire et pour les placettes témoins indiquent que d'une manière générale les $\mathrm{R}^{2}$ sont plus grands pour les placettes témoins que pour les placettes traitées. O'Hara (1988) constate également une plus faible corrélation entre l'accroissement en volume et la surface de la projection de cime chez le sapin Douglas après éclaircie. Il semble que cette baisse soit attribuable à la redistribution de l'espace de croissance et à l'absence de l'expansion de la cime verte (Brix 1981, O'Hara 1988), mais aussi au fait que l'éclaircie homogénéise la population d'arbres.

Le dhp de 1999 explique plus de $10 \%$ de variations en ig que le dhp initial de 1994. Le dhp relatif exprimant la position sociale 
Tableau 5. Coefficients de détermination $\left(R^{2}\right)$ du modèle ig $=\beta_{0}+\beta_{1} X$ pour l'accroissement quinquennal en surface terrière (ig) et les variables explicatives

\begin{tabular}{lccl}
\hline $\begin{array}{l}\text { Variable } \\
\text { explicative (x) }\end{array}$ & ig - témoin & ig - coupe & \\
\hline Dhp 1994 & 0,59 & 0,54 & \\
Dhp 1999 & 0,68 & 0,63 & Dimension de l'arbre \\
Hauteur totale 1999 & 0,40 & 0,39 & \\
\hline Dhp relatif & 0,73 & 0,50 & Position relative \\
\hline Hauteur de la cime & 0,42 & 0,43 & \\
Hc/ht & 0,25 & 0,31 & \\
Rayon de la cime & 0,71 & 0,67 & Cime verte \\
Surface de la cime & 0,66 & 0,61 & \\
Volume de la cime & 0,63 & 0,58 & \\
Projection de la cime & 0,70 & 0,66 & \\
Compression de la cime & 0,68 & 0,44 & \\
\hline Distance du voisin & 0,12 & 0,17 & Espace disponible \\
Dhp du voisin & 0,00 & 0,01 & \\
\hline
\end{tabular}

de l'arbre est plus fortement corrélé avec ig dans les placettes témoins que dans les placettes traitées, ce qui pourrait résulter de l'altération de la structure des placettes par la coupe (Marquis et Ernst 1991). On note également (Tableau 5) de fortes corrélations entre certaines variables de la cime verte et l'accroissement quinquennal en surface terrière. En effet, le rayon, la projection, l'aire latérale, le volume et la compression de la cime verte présentent de fortes corrélations avec ig, ce qui confirme les observations des autres (Assmann 1970, Frank 1973, Athari et Kramer 1988, O’Hara 1988, Larocque et Marshall 1994) à l'effet que les dimensions de la cime verte ont une valeur informationnelle importante pour évaluer la vigueur et la réaction des arbres au traitement sylvicole. Il faut rappeler cependant que si l'ensemble des relations significatives entre l'accroissement quinquennal en surface terrière sont positives, celle entre ig et la compression latérale de la cime est négative.

Les relations entre l'accroissement quinquennal en surface terrière et le ratio de la cime vivante, la distance du voisin et le dhp du voisin sont faibles ou négligeables (Tableau 5). Cette dernière observation est en accord avec les résultats obtenus par Cole et Lorimer (1994) pour l'érable à sucre (Acer saccharum), le frêne d'Amérique (Fraxinus americana) et le tilleul d'Amérique (Tilia americana). Elle s'expliquerait par le fait que la distance et la grosseur du plus proche voisin sont des estimateurs indirects moins précis de l'espace de croissance et du statut d'un arbre que les mesures de la cime verte. À la vue de ces résultats, la prescription de la coupe préparatoire (nature de l'intervention) dans ce type de peuplement devrait tenir compte de l'état de la cime verte afin de maximiser les effets positifs sur la croissance radiale.

\section{Intensité de coupe et accroissement en surface terrière des placettes}

La relation entre l'intensité de la coupe et l'accroissement en surface terrière a été évaluée par la régression multiple. Considérant que la coupe a été appliquée au niveau de la placette, les variables indépendantes sont l'intensité de coupe (int), soit la surface terrière coupée sur la surface terrière avant la coupe et le dhp moyen $(\bar{d})$ des arbres échantillonnés en 1994 après la coupe. Les variables dépendantes sont :

1) l'accroissement moyen en surface terrière ( $\mathrm{ig}_{\mathrm{AP}}$ ) des arbres échantillonnés de la placette entre 1995 et 1999;
2) le ratio moyen de l'accroissement en surface terrière des arbres échantillonnés de la placette de 1995 et 1999 sur l'accroissement en surface terrière des arbres échantillonnés de 1990 et $1994\left(\mathrm{ig}_{\mathrm{AP}} / \mathrm{ig}_{\mathrm{AV}}\right)$;

3) le rapport moyen entre $\mathrm{ig}_{\mathrm{AV}}$ et la surface de projection de la cime verte des arbres échantillonnés (ig/PCV).

\section{Accroissement quinquennal en surface terrière - $\mathrm{ig}_{\mathrm{AP}}$}

La régression multiple permet de conclure à une relation très étroite entre l'accroissement quinquennal en surface terrière, le dhp de 1994 et l'intensité de la coupe ( $\mathrm{p}<0,0001)$. Le modèle de régression multiple [4] permet d'expliquer $86 \%$ des variations en ig $_{\mathrm{AP}}$.

$$
\text { ig }_{\mathrm{AP}}=-0,508+0.047 \cdot \bar{d}+0,003 \text {.int }
$$

La contribution de deux variables indépendantes est significative $(\mathrm{p}<0,0001)$ pour le dhp et $\mathrm{p}=0,005$ pour l'intensité de coupe) et elle s'élève à $74 \%$ pour le dhp de $1994(\bar{d})$ et à $12 \%$ complémentaires pour l'intensité de coupe. Ces résultats suggèrent que le dhp et la coupe ont un effet significatif sur l'accroissement quinquennal en surface terrière. Le lien entre le dhp et ig est linéaire (Fig. 3) et pour bien comprendre cette relation en ce qui a trait à l'arbre, nous avons reproduit les données de l'accroissement en surface terrière et du dhp relatif des arbres échantillonnés dans les placettes éclaircies à plus de $40 \%$ (Fig. 4). On constate que les arbres dont la taille relative est supérieure (la partie droite du graphique) sont plus productifs que les petits arbres occupant une position sociale inférieure (la partie gauche de graphique).

En ce qui concerne la coupe, un t-test nous permet de conclure $(\mathrm{p}<0,0001)$ que son effet sur l'accroissement quinquennal en surface terrière est plus important (Fig. 6) lorsque son intensité est supérieure à $30 \%$. De plus, un t-test nous permet de conclure que les placettes témoins et les placettes à intensité de coupe faible ne présentent pas d'accroissements quinquennaux en surface terrière différents $(p=0,414)$ avec une moyenne de $0,23 \mathrm{dm}^{2}$. On en vient à la même conclusion lorsqu'on compare les placettes à intensités de coupe moyenne et forte $(p=0,666)$ avec un accroissement moyen de $0,42 \mathrm{dm}^{2}$ par arbre. À titre de comparaison, l'accroissement moyen quinquennal par arbre selon les tables de Vézina et Linteau (1968) pour la $1^{1 \text { ère }}$ classe entre 55 et 60 ans est de $0,31 \mathrm{dm}^{2}$.

La faible productivité des arbres de la strate sociale inférieure, qui confirme les conclusions de Assmann (1970), est sans doute causée par les cimes chétives, fortement comprimées et dont le maintien de la respiration est très coûteux (Gilmore et Seymore 1996). Malgré une forte plasticité morphologique de la cime du sapin (Parent et Messier 1995), la position sociale et la densité des placettes ne lui permettent pas de déployer latéralement les branches afin de maximiser l'interception de la lumière et de réduire l'entretien coûteux des structures non photosynthétiques (Sprugel 1989, Waring 1991, Roberts et Long 1992).

\section{Ratio ig AP $_{\text {Aig }}$ AV}

La régression multiple entre le ration $\mathrm{ig}_{\mathrm{AP}} / \mathrm{ig}_{\mathrm{AV}}$ et les variables indépendantes ( $d$ et int) permet de conclure à une relation significative $(p=0,0008)$. Cependant, la contribution $d u$ dhp de 1994 dans le modèle ne l'est pas $(\mathrm{p}=0,822)$. Ce résultat suggère que l'augmentation du rendement en surface terrière par rapport à la période précédente ne dépend que de l'intensité de coupe. 

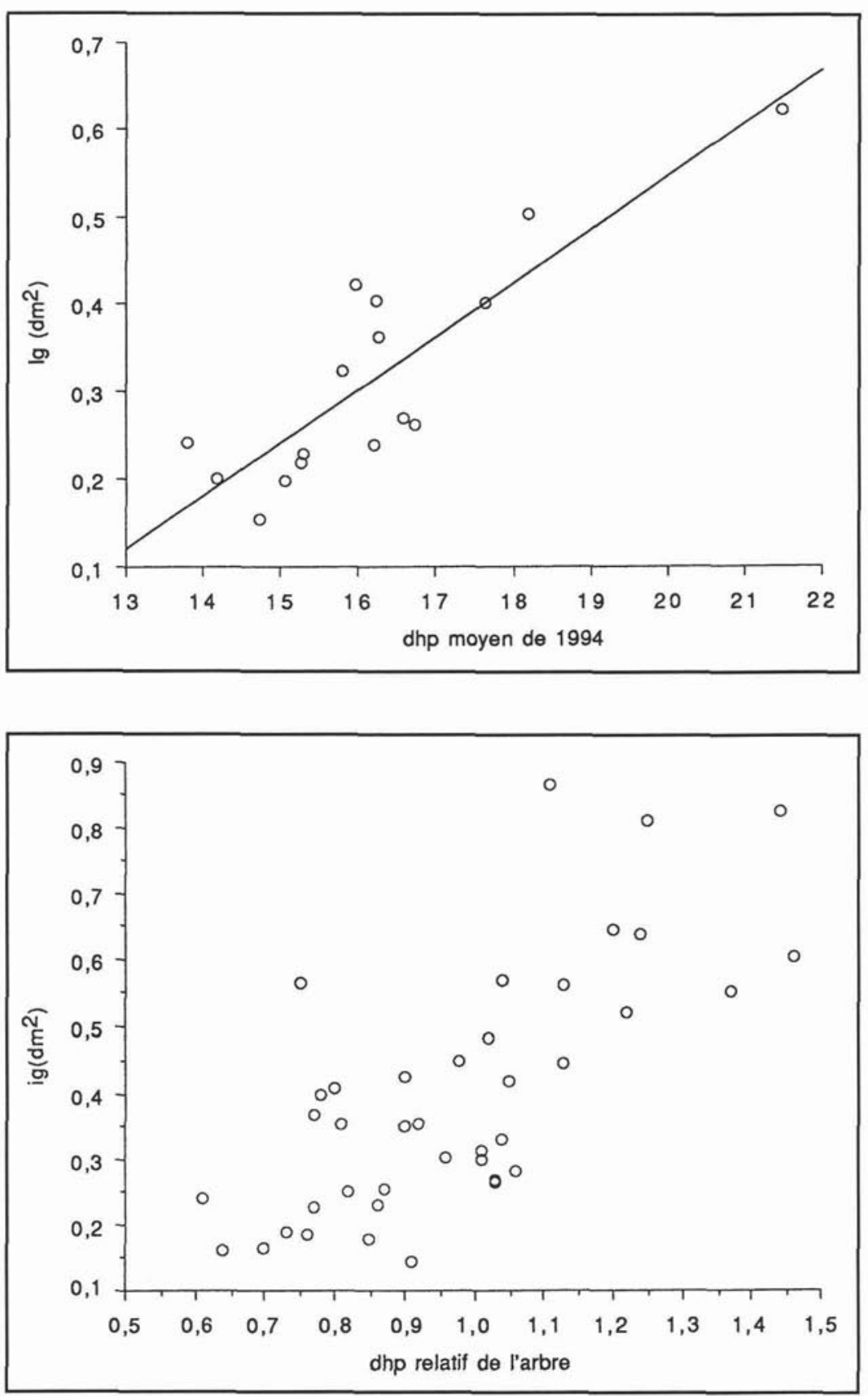

Fig. 3. Accroissement moyen quinquennal en surface terrière selon le dhp moyen des placettes de 1994 .

Fig. 4. Accroissement quinquennal en surface terrière des arbres échantillons en fonction du dhp relatif.
L'étude du ratio selon l'intensité de coupe (Fig. 6) indique une relation non linéaire hautement significative $(p<0,0001)$. Le modèle [5] utilisant le carré de l'intensité de coupe comme variable dépendante explique $71 \%$ des variations du ratio entre l'accroissement en surface terrière au cours des 5 dernières années par rapport à la période précédente :

$$
\text { ratio ig } \mathrm{AP}_{\mathrm{Ag}} / \mathrm{ig}_{\mathrm{AV}}=1,436+0,000439 \mathrm{int}^{2}
$$

Par ailleurs, un t-test permet de conclure que les placettes témoins et faiblement éclaircies produisent un ratio (entre l'accroissement en surface terrière au cours des 5 dernières années par rapport à la période précédente) significativement plus faible (p $<0,0001)$ que les placettes éclaircies avec des intensités moyenne et forte. Ces résultats suggèrent que l'intensité de coupe doit être plus grande que $30 \%$ pour susciter une augmentation significative du ratio par rapport aux témoins. La réaction des placettes à la coupe montre que le peuplement a encore la capacité de réagir au traitement intense, ce qui est en accord avec les tables de Vézina et Linteau (1968). Par contre, nos résultats constrastent avec les courtes révolutions préconisées pour les sapinières par Boudoux (1978) et Bolghari (1989).

\section{Accroissement en surface terrière selon la projection de la cime verte}

Enfin, la régression entre les ratios $\mathrm{ig}_{\mathrm{AP}} / \mathrm{PCV}$ et les variables indépendantes (dhp moyen de 1994 et intensité de coupe) permet de conclure à une relation non significative $(p=0,142)$. Ce résultat suggère que ni la coupe ni la grosseur initiale de l'arbre n'ont d'effet sur l'efficacité de la production du sapin baumier 

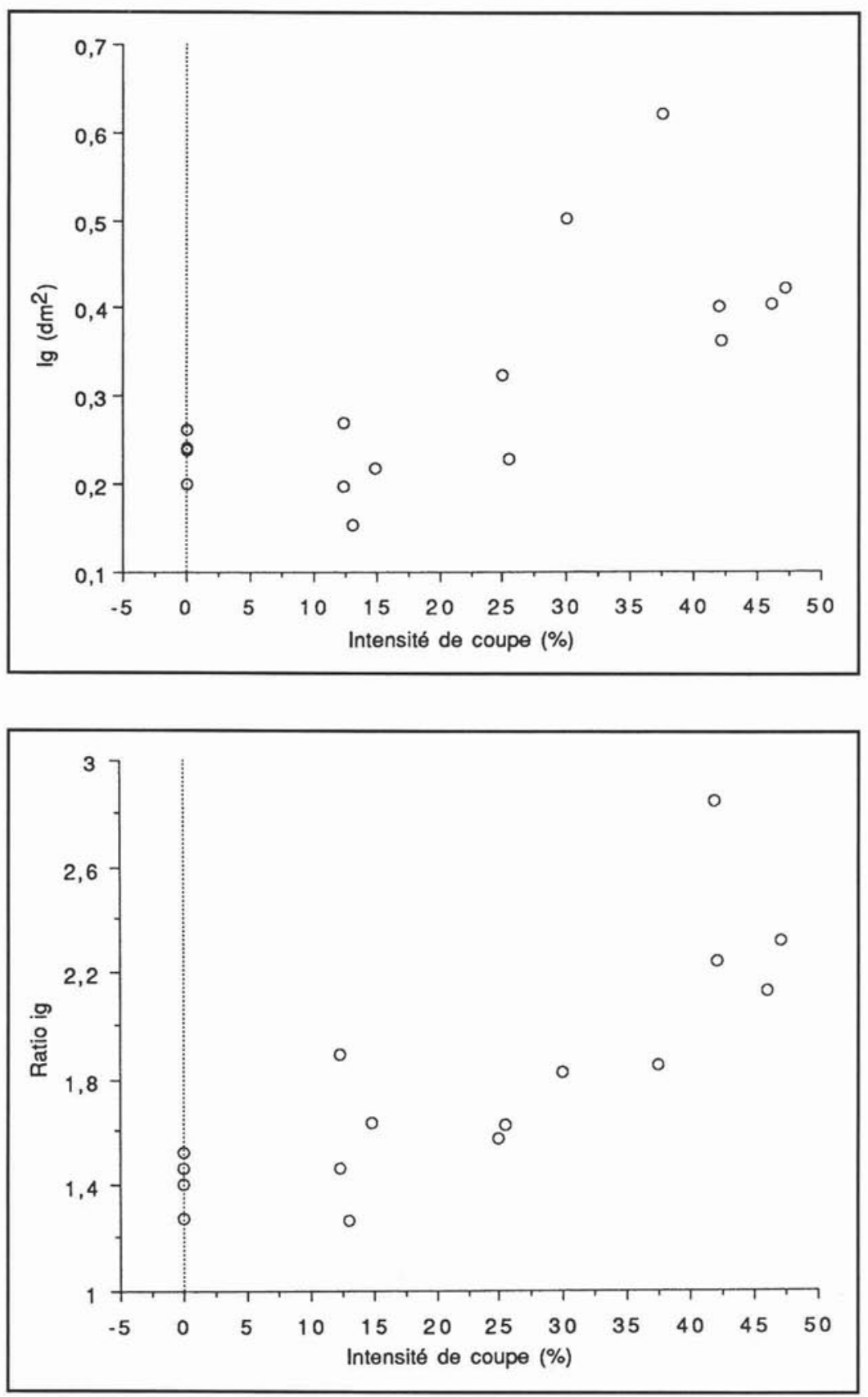

Fig. 5. Accroissement moyen quinquennal des placettes en surface terrière selon l'intensité de coupe.
Fig. 6. Ratio de l'accroissement moyen quinquennal en surface terrière après la coupe $\left(\mathrm{ig}_{\mathrm{AP}}\right)$ sur celui avant la coupe $\left(\mathrm{ig}_{\mathrm{AV}}\right)$ des placettes selon l'intensité de coupe. et par conséquent sur la surface terrière produite depuis le traitement par mètre carré de la cime verte projetée. Autrement dit, l'efficacité de la production des structures photosynthétiques ne dépend que de l'importance de la cime verte.

\section{Conclusions}

Dans le cadre de la présente étude, les résultats obtenus montrent que la coupe préparatoire appliquée dans le dispositif expérimental a stimulé la croissance des arbres résiduels par une augmentation de leur diamètre moyen. La mortalité observée dans le dispositif ne semble pas être influencée par l'intensité de la coupe.

La coupe préparatoire a provoqué des modifications dans la hiérachie et dans la structure des placettes. De par la sélection des tiges à couper, cette coupe a affecté la compression moyenne de la cime verte du sapin baumier. La compression diminue avec l'intensité du traitement et avec la taille croissante de l'arbre.

Les résultats obtenus pour le sapin baumier du dispositif expérimental indiquent de fortes corrélations entre l'accroissement en surface terrière, la dimension de l'arbre et la position relative de l'arbre dans le peuplement et certaines variables de la cime verte.

La coupe préparatoire a eu un effet significatif sur l'accroissement quinquennal en surface terrière et en particulier lorsque son intensité était plus grande que $30 \%$. La réaction des arbres aux dph relatifs supérieurs est plus importante que la réaction des petits arbres. La réaction positive du sapin au traitement indique qu'il est possible d'envisager de prolonger l'âge de l'exploitabilité absolue pour un peuplement de seconde venue de 60 ans. 


\section{Remerciements}

Nous remercions MM. Gilles Bélanger et Roger Gagné pour la prise des données sur le terrain, ainsi que MM. Gilles Tessier et Romain Chénard pour la coupe du bois. Enfin, nous remercions M. Guy R. Larocque et deux lecteurs anonymes pour les critiques constructives du manuscrit.

\section{Références}

Assmann, E. 1970. The Principles of Forest Yield Study. Pergamon Press, New York. 506 p.

Athari, S. et H. Kramer. 1988. Beziehungen zwischen Grundflächenzuwachs und verschiedenen Baumparameterns in geschädigten Buchenbeständen. Algemeine Forst und Jagd-Zeitung. 160(5): 77-83. Baldwin, V.C. 1977. Regeneration following shelterwood cutting in a New Brunswick softwood stand. Maritimes Forest Research Centre, Canadian Forestry Service, Inf. Rep. M-X-76, 23 p.

Bolghari, H.A. 1989. Aperçu sur les traitements d'éclaircie et de dégagement dans les sapinières. Dans Ordre des ingénieurs forestiers du Québec et Université Laval (Éd.) Colloque sur la sapinière et son aménagement, Forêt Montmorency, Québec, 21 et 22 septembre 1989.

Boudoux, M. 1978. Tables de rendement empiriques pour l'épinette noire, le sapin baumier et le pin gris au Québec. Gouvermement du Québec, ministère des Terres et forêts et Gouvernement du Canada, Service canadien des forêts. $101 \mathrm{p}$.

Brix, H. 1981. Effects of thinning and nitrogen fertilization on branch and foliage production in Douglas-fir. Can. J. For. Res. 11: 502-511.

Burschel, P. et J. Huss. 1997. Grundriss des Waldbaus. Ein Leitfaden für Studium und Praxis. Parey Buchverlag, Berlin. 497 p.

Cole, W.G. et C.G. Lorimer. 1994. Predicting tree growth from crown variables in managed northern hardwood stands. For. Ecol. Manag. 67: 159-175.

Corriveau, A. 1971a. L'intensité de l'éclaircie, ses effets sur un peuplement de sapin baumier de 40 ans. Ministère de l'Environnement, Service canadien des forêts, Rapp. Inf. Q-F-X-20. 43 p.

Corriveau, A. 1971b. Étude comparative de coupes partielles effectuées en 1954 dans les peuplements de sapin baumier de différents âges de la rivière Épaule, Québec. Ministère de l'Environnement, Service canadien des forêts, Rapp. Inf. Q-F-X-21. 33 p.

Dong, P.H. et H. Kramer. 1986. Kronenparameter zur Schadensermittlung in Nadelholzbeständen. Proceedings of an International Conference. Environmental influences on tree and stand increment. September 23-27, 1985. Durham, New Hampshire, USA.

Fienberg, S.E. 1980. The analysis of cross-classified categorical data. Second Edition. MIT Press, Cambridge, MA. 198 p.

Frank, R.M. 1973. The course of growth response in released white spruce: 10-year results. USDA Forest Service Research Paper NE-258. $6 \mathrm{p}$.

Frank, R.M. 1990. Abies balsamea - balsam fir. Dans R.M. Burns and B.H. Honkala (eds.) Silvics of North America. Vol. 1. Conifers. pp. 26-35. U.S. Dep. Agric. Agric. Handb. 654.

Gilmore, D.W. et R.S. Seymore. 1996. Alternative measures of stem growth efficiency applied to Abies balsamea from four canopy positions in central Maine, USA. For. Ecol. Manag. 84: 209-218.

Grandtner, M.M. 1966. La végétation forestière du Québec méridional. Les Presses de l'Université Laval, Québec. 216 p.

Hannah, P.R. 1988. The shelterwood method in northeastern forest types: A literature review. North. J. Appl. For. 5: 70-77.

Hatcher, R.J. 1961. Partial cutting of balsam fir stands on the Epaule River watershed, Quebec. Department of Forestry, Forest Research Branch Tech. Note No. 105. 29 p.

Hatcher, R.J. 1963. Effects of birch dieback and spruce budworm on forest development. Forest section L.6, Quebec. Dept. of Forestry Publication No. 1014. 16 p.

Horne, R., G. Robinson et J. Gwalter. 1986. Response increment: A method to analyze thinning response in even-aged forests. For. Sci. 32: $243-253$.
Johnston, W.F. 1986. Manager's handbook for balsam fir in North Central States. USDA For. Serv. Gen. Tech. Rep. NC-111.

Lanier, L. 1986. Précis de sylviculture. École nationale du génie rural, des eaux et des forêts, Nancy. 468 p.

Larocque, G.R. et P.L. Marshall. 1994. Crown development in red pine stands. II. Relationships with stem growth. Can. J. For. Res. 24: 775-784.

Lavigne, M.B. et J.G. Donnelly. 1989. Early results of a spacing trial in a precommercially thinned balsam fir stand in western Newfoundland. Forestry Canada, Newfoundland and Labrador Region, St. John's. Nfld. Inf. Rep. N-X-269. 23 p.

Linteau, A. 1957. Classification des stations forestières de la section des conifères du nord-est, région forestière boréale du Québec, ministère du Nord canadien et des Ressources nationales, Service forestier Divison des recherches sylvicoles, Bulletin No. 118. 193 p. Marquis, D.A. et R.L. Ernst. 1991. The effects of stand structure after thinning on the growth of an Allegheny hardwood stand. For. Sci. 37: 1182-1200.

Matthews, J.D. 1994. Silviculture systems. Clarendon Press, Oxford. $284 \mathrm{p}$.

Noêl, C. et R. Rouleau. 1979. Parcelles-échantillons permanentes. Normes techniques. Ministère de l'Énergie et des Ressources du Québec, Service de l'inventaire forestier.

O'Hara, K.L. 1988. Stand structure and growing space efficiency following thinning in an even-aged Douglas-fir stand. Can. J. For. Res. 18: 859-866.

Parent, S. et C. Messier. 1995. Effets d'un gradient de lumière sur la croissance en hauteur et la morphologie de la cime du sapin baumier régénéré naturellement. Can. J. For. Res. 25: 878-885.

Proulx, H., G. Jacques, A.M. Lamothe et J. Litynski. 1987. Climatologie du Québec méridional. Ministère de l'Environnement du Québec, Direction de la Météorologie, Québec, M.P. 65. 198 p.

Raymond, P. 1998. Efficacité du système de régénération par coupes progressives dans les sapinères boréales riches : résultats cinq ans après la coupe d'ensemencement. Thèse de maîtrise, Université Laval.

Roberts, S.D. et J.N. Long. 1992. Production efficiency of Abies lasiocarpa: influence of vertical distribution of leaf area. Can. J. For. Res. 22: $1230-1234$.

Sprugel, D.G. 1989. The relationship of evergreenness, crown architecture, and leaf size. Am. Nat. 133: 465-479.

SPSS Inc. 1998. SYSTAT ${ }^{\circledR}$ 8.0: Statistics. Chicago, IL. 1086 p.

Steneker, G.A. 1974. Selective cutting to release white spruce in 75 to 100-year-old white spruce-trembling aspen stands, Saskatchewan. Canadian Forestry Service, Northern Forestry Centre, Edmonton, Alta. Inf. Rep. NOR-X-121.

Thibault, M. 1986. Les régions écologiques du Québec méridional (deuxième approximation). Carte synthèse. Ministère de l'Énergie et des Ressources du Québec, Service de la recherche et Service de cartographie, Québec, Canada.

Vézina, P.E. et A. Linteau. 1968. Growth and yield of balsam fir and black spruce in Quebec. Canadian Department of Forestry and Rural Development, Forestry Branch, Forest Research Laboratory, SainteFoy, Québec. Inf. Rep. Q-X-2.

Waring, R.H. 1991. Responses of evergreen trees to multiple stresses. Dans H.A. Mooney, W.E. Winner and E.J. Pell (Eds.). Response of plants to multiple stresses. pp 371-387. Academic Press, New York. Youngblood, A.P. 1990. Radial growth after a shelterwood seed cut in a mature stand of white spruce in interior Alaska. Can. J. For. Res. 21: $410-413$.

Yuill, B.B. 1980. Shelterwood harvesting. Scott Paper Int. Inc., Valley District, Truro, Nova Scotia, Office Report 21.26 p.

Zarnovican, R. 1982. Examen de quelques relations allométriques chez le sapin baumier et l'épinette blanche. Can. J. For. Res. 12: 171-180. Zarnovican, R. 1998. Observations sur le chablis et la carie dans une sapinière de seconde venue dans la forêt modèle du Bas-Saint-Laurent. Ressources naturelles Canada, Service canadien des forêts, Centre de foresterie des Laurentides, Sainte-Foy, Qc. Notes de recherche No. 3. 2 p. 
Zarnovican, R. et C. Laberge. 1996. Effet de l'éclaircie précommerciale sur la production de jeunes sapinières de la Haute-Côte-Nord. Ressources naturelles Canada, Service canadien des forêts, Région du Québec, Sainte-Foy, Qc. Rapp. Inf. LAU-X-118.
Zarnovican, R. et C. Laberge. 1998. Radial growth of balsam fir [Abies balsamea (L.) Mill.] associated with past spruce budworm [Choristoneura fumiferana (Clem.)] outbreaks in Quebec, Canada. Lesnictvi-Forestry 44(11): 481-487. 